\title{
Multi-Area Unit Scheduling and Reserve Allocation Under Wind Power Uncertainty
}

\author{
Ali Ahmadi-Khatir, Member, IEEE, Antonio J. Conejo, Fellow, IEEE, and Rachid Cherkaoui, Senior Member, IEEE
}

\begin{abstract}
This paper proposes a decentralized methodology to optimally schedule generating units while simultaneously determining the geographical allocation of the required reserve. We consider an interconnected multi-area power system with cross-border trading in the presence of wind power uncertainty. The multi-area market-clearing model is represented as a two-stage stochastic programming model. The proposed decentralized procedure relies on an augmented Lagrangian algorithm that requires no central operator intervention but just moderate interchanges of information among neighboring regions. The methodology proposed is illustrated using an example and a realistic case study.
\end{abstract}

Index Terms-Decentralized unit scheduling, interconnections, multi-area power system, reserve allocation, wind power.

\section{INTRODUCTION}

\section{A. Motivation and Aim}

$\mathbf{T}$ HE increasing integration of regional and national electric energy systems will undoubtedly accelerate interregional trading (in both energy and reserve) and increase the importance of coordinating unit scheduling and reserve allocation among interconnected electricity markets.

Moreover, the large-scale integration of wind generation into power systems presents a significant challenge to system operators due to the unpredictable and highly variable nature of wind power generation. Hence, it is important to develop marketclearing models that ensure a secure and economically efficient operation of each regional/national system and of the interconnected system as a whole under and in spite of uncertainties.

The reserve is the commodity traded in the market to counteract unpredictable changes in the system conditions. Traditional rules for the determination of reserve services have worked effectively in practice for thermal-dominated system. Typically, these rules are based on the deterministic $n-1$ criteria, which guarantee the appropriate operation of the system in the case of outage of one generating unit or a major transmission line. As the penetration of wind power capacity increases, the need of reserve services to cover net

Manuscript received May 12, 2013; revised August 01, 2013 and October 16, 2013; accepted November 27, 2013. Date of publication December 19, 2013; date of current version June 16, 2014. This work was supported by Swiss Electric Research for the project of multi-area security assessment. Paper no. TPWRS-00590-2013.

A. Ahmadi-Khatir and R. Cherkaoui are with the Power System Research Group, Ecole Polytechnique Federale de Lausanne (EPFL), Lausanne, Switzerland (e-mail: ali.khatir@epfl.ch; rachid.cherkaoui@epfl.ch).

A. J. Conejo is with the Universidad de Castilla-La Mancha, Ciudad Real, Spain (e-mail: antonio.Conejo@uclm.es).

Digital Object Identifier 10.1109/TPWRS.2013.2293542 demand fluctuations increases. On the other hand, as the level of inter-regional trading grows, the reserve services required for the whole interconnected system may decrease thanks to sharing. As a result of the features described above, traditional deterministic rules are generally inadequate in the presence of large-scale integration of wind generation and/or high inter-regional trading, since such rules do not consider the stochastic nature of wind power generation and the reserve assistance from neighboring areas, thus calling for a more sophisticated approach for reserve determination. Additionally, note that traditional rules generally result in either over-protection or under-protection of the considered system.

Ideally, a central operator with access to all data (including technical and economical data) of the whole system, could centrally operate the system, but for many political and technical reasons, this kind of operator is unlikely to be implemented. Hence, the multi-regional electricity markets are generally operated by the coordinated actions of regional system operators. In fact, such coordinated model allows the market/system operator (MO/ISO) of each interconnected area to clear its market, i.e., to find out the optimal unit scheduling and reserve allocation, independently of other areas, while interchanging some border information of its system with the adjacent areas.

The focus of this paper is to provide a methodology to optimally schedule generating units (unit commitment) and to determine the geographical allocation and levels of the required reserves taking into account inter-regional transmission line constraints. In particular, the methodology is based on a decentralized procedure for clearing multi-regional electricity markets, formulated as a two-stage stochastic programming problem, under wind power uncertainty. The objective is to maximize the overall expected social welfare while preserving scheduling independence of the involved areas.

The proposed market-clearing model is motivated by multiregional electricity markets in the U.S. (e.g., PJM interconnection [1]) where the unit commitment problem is considered to clear the market. Note that including $0 / 1$ binary variables, representing the on/off status of generating units, into the marketclearing problem makes it non-convex and thus significantly more complex.

The proposed decentralized algorithm relies on a relaxation technique based on the augmented Lagrangian and requires interchanges of moderate information among neighboring regions [2]. This decomposition scheme is not oriented to improve the computational efficiency, but rather to preserve the independence of each area in a multi-area power system. In fact, what our paper does is providing an approach to operate a system with significant interconnections and wind integration in a decentralized manner, and fully preserving the advantage of the interconnections. 


\section{B. Literature Review and Contributions}

Using stochastic programming to deal with uncertainty in the market-clearing problem (i.e., unit scheduling and reserve allocation) in a single-area power system is reported, for instance, in [3]-[8]. However, this subject has not been thoroughly investigated in multi-area system. Nonetheless, several works considering fully reliable multi-area systems (i.e., uncertainty free) have been reported in the literature (e.g., [9]-[13]). These works differ in terms of the decoupling principles and decomposition algorithms.

In [9] and [10] the decoupling is carried out considering a fictitious border bus inside an overlapping zone between two neighboring areas in such a way that all duplicated variables pertaining to this fictitious bus are forced to be equal at the optimum. In [11] the decoupling is achieved by including one or two fictitious buses per interconnecting line, and the power balance equations of the fictitious buses are included as coupling constraints into the original problem. The coupling constraints in [12] and [13] are the power flow and the capacity limit equations of the tie-lines.

Applications of the augmented Lagrangian relaxation to solve a multi-area OPF in a decentralized manner are described in [9] and [10], while in [11] a standard Lagrange relaxation is used. References [12] and [13] use the optimality condition decomposition algorithm to solve the multi-area OPF problem.

Reference [14] presents a stochastic market-clearing model for a multi-area power system. The model described in [14] corresponds to an auction inspired by those used by most European electricity markets, which leave the on/off commitment decisions to the producers that own the production units.

All the above references address the OPF problem (i.e., they do not include unit commitment $0 / 1$ variables in the model). We have found no reference in the domain of decentralized multi-area stochastic unit commitment. Hence, we propose to extend the market-clearing procedure presented in [14] by incorporating unit commitment $0 / 1$ variables in the formulation. Note that including $0 / 1$ variables make the model non-continuous and thus non-convex, and therefore much harder to solve. In order to solve the proposed model in a decentralized manner, a decomposition algorithm different than the one proposed in [14] is required as the decomposition algorithm in [14] requires continuity [15].

The procedure proposed in this paper relies on the decoupling principle reported in [11] and on an augmented Lagrange relaxation technique for fully exploiting the structure of a multiarea stochastic unit commitment problem to achieve single-area problems that can be solved independently. Hence, within the above framework, the main contribution of this paper is developing an appropriate mathematical tool based on stochastic programming to assist the system operators of a multi-area power system to optimally schedule generating units and to allocate the reserve required in a decentralized manner under wind power uncertainty and precisely representing interactions among areas.

\section{Paper Organization}

The rest of the paper is organized as follows. Section II is the core of the paper providing the model assumption (Section II-B), the mathematical formulation of the market-clearing model in a centralized form (Section II-C), the area decoupling principle (Section II-D), and the mathematical formulation in a decentralized form (Section II-E). Section II-F discusses convergence properties of the decomposition algorithm, the information required to be interchanged among areas and how this algorithm can be implemented in practice. Section III reports and discusses results obtained from a two-area three-bus test system and the three-area IEEE-RTS. The conclusions drawn from this study are given in Section IV.

\section{Multi-Area Unit Scheduling Model}

\section{A. Notation}

The notation is provided below.

1) Indices and Sets:

\begin{tabular}{|c|c|}
\hline$A, B$ & Indices of areas. \\
\hline$i$ & Index of generating units in area $\mathrm{A}$, from 1 to $N_{\mathrm{G}}^{A}$. \\
\hline$j$ & Index of wind plants in area $\mathrm{A}$, from 1 to $N_{\mathrm{J}}^{A}$. \\
\hline$n, m, r$ & $\begin{array}{l}\text { Indices of buses and loads in area A, from } 1 \text { to } \\
N_{\mathrm{B}}^{A} \text {. }\end{array}$ \\
\hline$w$ & Index of wind scenarios, from 1 to $N_{\mathrm{W}}$. \\
\hline$t$ & Index of time periods, from 1 to $N_{\mathrm{T}}$. \\
\hline$\Lambda_{A}$ & Set of internal lines of area A. \\
\hline$\Gamma_{A}$ & Set of tie-lines of area A. \\
\hline$\Phi_{n}^{\mathrm{G}, \mathrm{A}}$ & Set of generating units at bus $n$ of area A. \\
\hline$\Phi_{n}^{\mathrm{W}, \mathrm{A}}$ & Set of wind plants at bus $n$ of area A. \\
\hline$\Psi_{n}^{A}$ & $\begin{array}{l}\text { Set of external buses in neighboring areas } \\
\text { connected to border bus } n \text { in area A. }\end{array}$ \\
\hline$F^{A}$ & Set of fictitious buses of area A. \\
\hline$\Omega_{n}^{A}$ & Set of buses connected to interior bus $n$ in area A. \\
\hline$\Delta_{n}^{A}$ & $\begin{array}{l}\text { Set of buses (including interior, border and } \\
\text { fictitious buses in area A) connected to bus } n \text { in } \\
\text { area A. }\end{array}$ \\
\hline$\Pi_{z^{A}}^{A}$ & $\begin{array}{l}\text { Set of border buses in area A connected to } \\
\text { fictitious bus } z^{A} \text { of area A. }\end{array}$ \\
\hline
\end{tabular}

2) Parameters:

$C_{i t}^{\mathrm{SU}} \quad$ Start-up cost of unit $i$ in period $t[\$]$.

$d_{t} \quad$ Duration of time period $t[\mathrm{~h}]$.

$q_{i t}^{\mathrm{D}} \quad$ Price offer of unit $i$ for downward reserve at time $t$ [\$/MWh].

$q_{i t}^{\mathrm{U}} \quad$ Price offer of unit $i$ for upward reserve at time $t$ [\$/MWh].

$f_{n r}^{A, \max } \quad$ Maximum capacity of internal or tie-line $n r$ of area $A[\mathrm{MW}]$.

$L_{n t}^{A} \quad$ Load demand of bus $n$ of area $A$ at time $t$ [MW].

$P_{i}^{\max } \quad$ Capacity of unit $i[\mathrm{MW}]$.

$P_{i}^{\min } \quad$ Minimum power output of unit $i$ [MW].

$P_{j t}^{\text {Offer }} \quad$ Wind power forecast of wind plant $j$ in period $t$ as offered in the market [MW]. 
$P_{j w t}^{W} \quad$ Realization of wind power associated with wind plant $j$ in period $t$ and scenario $w$ [MW].

$R_{i}^{\mathrm{D}, \max } \quad$ Maximum downward reserve can be provided by unit $i[\mathrm{MW}]$.

$R_{i}^{\mathrm{U}, \max } \quad$ Maximum upward reserve can be provided by unit $i[\mathrm{MW}]$.

$R D_{i}^{\text {down }} \quad$ Ramp-down rate limit of unit $i$ [MW].

$R U_{i}^{\text {up }} \quad$ Ramp-up rate limit of unit $i$ [MW].

$x_{n r} \quad$ Reactance of the line between buses $n$ and $r$ [per unit].

$\pi_{w} \quad$ Weight of scenario $w$.

$\nu_{n t}^{\text {Lost }} \quad$ Value of lost load for demand $n$ in period $t$ [\$/MWh].

\section{3) Variables:}

$L_{n w t}^{\text {shed }}$

Load shedding imposed on load $n$ of area A in period $t$ and scenario $w$ [MW].

$P_{i t}^{\mathrm{G}} \quad$ Power scheduled for unit $i$ in period $t$ at the market stage [MW].

$P_{j t}^{W} \quad$ Wind power scheduled for wind plant $j$ in period $t$ at the market stage [MW].

$P_{j w t}^{\text {spill }} \quad$ Wind power spillage of wind plant $j$ in period $t$ and scenario $w$ [MW].

$R_{i t}^{\mathrm{D}} \quad$ Downward reserve scheduled for unit $i$ at time $t$ [MW].

$R_{i t}^{\mathrm{U}} \quad$ Upward reserve scheduled for unit $i$ in period $t$ [MW].

$r_{i w t}^{\mathrm{D}} \quad$ Downward reserve deployed by unit $i$ at time $t$ and scenario $w[\mathrm{MW}]$.

$r_{i w t}^{\mathrm{U}} \quad$ Upward reserve deployed by unit $i$ in period $t$ and scenario $w$ [MW].

$u_{i t} \quad$ Binary variable that is equal to 1 if unit $i$ is on in period $t$ and 0 otherwise.

$y_{i t} \quad$ Variable that is equal to 1 if unit $i$ is started up at the beginning of period $t$ and 0 otherwise.

$\theta_{n t}^{A} \quad$ Phase angle of bus $n$ of area A in period $t[\mathrm{rad}]$.

$\delta_{n w t}^{A} \quad$ Phase angle of bus $n$ of area $\mathrm{A}$ in period $t$ and scenario $w$ [rad].

\section{B. Model Assumption}

Pursuing clarity and simplicity, the main modeling assumptions considered throughout this paper are listed below.

1) Loads are assumed to be inelastic and they do not participate in the reserve market.

2) A lossless dc representation of the transmission network is embedded within the considered model.

3) Wind power producers offer their forecast productions at zero price.

4) The supply cost functions of thermal generating units are described by quadratic functions, i.e., $C_{i}\left(P_{i t}^{\mathrm{G}}\right)=c_{i}\left(P_{i t}^{\mathrm{G}}\right)^{2}+b_{i} P_{i t}^{\mathrm{G}}+a_{i}$.
5) Minimum up- and down-time constraints of thermal units are not considered; the unit shutdown costs are assumed negligible. Nonetheless, such inter-temporal constraints of generating units can be easily included in the market-clearing procedure [16].

6) Only wind power uncertainty is included into the model.

7) The operators of interconnected areas need to agree on the wind scenarios to be considered to clear the market as they need to interchange information per scenario.

8) Non-spinning reserve is not considered.

Note that most of the above simplifications are easily removed.

\section{Centralized Multi-Area Unit Scheduling Formulation}

The centralized model is characterized as stated below.

Minimize

$$
\begin{aligned}
\sum_{A}\left\{\sum_{t=1}^{N_{\mathrm{T}}} \sum_{i=1}^{N_{\mathrm{G}}^{A}} y_{i t} C_{i t}^{S U}\right. \\
\quad+\sum_{t=1}^{N_{\mathrm{T}}} d_{t}\left[\sum_{i=1}^{N_{\mathrm{G}}^{A}} C_{i}\left(P_{i t}^{\mathrm{G}}\right)+\sum_{i=1}^{N_{\mathrm{G}}^{A}}\left(q_{i t}^{\mathrm{U}} R_{i t}^{\mathrm{U}}+q_{i t}^{\mathrm{D}} R_{i t}^{\mathrm{D}}\right)\right] \\
\quad+\sum_{w=1}^{N_{\mathrm{W}}} \pi_{w} \sum_{t=1}^{N_{\mathrm{T}}} d_{t}\left[\sum_{i=1}^{N_{\mathrm{G}}^{A}}\left(C_{i}\left(P_{i t}^{\mathrm{G}}+r_{i w t}^{\mathrm{U}}-r_{i w t}^{\mathrm{D}}\right)-C_{i}\left(P_{i t}^{\mathrm{G}}\right)\right)\right] \\
\left.\quad+\sum_{w=1}^{N_{\mathrm{W}}} \pi_{w} \sum_{t=1}^{N_{\mathrm{T}}} d_{t}\left[\sum_{n=1}^{N_{\mathrm{B}}^{A}} \nu_{n t}^{\mathrm{Lost}} L_{n w t}^{\text {shed }}\right]\right\}
\end{aligned}
$$

subject to

$$
\begin{gathered}
\left\{\sum_{i \in \Phi_{n}^{\mathrm{G}, \mathrm{A}}} P_{i t}^{\mathrm{G}}+\sum_{j \in \Phi_{n}^{\mathrm{W}, \mathrm{A}}} P_{j t}^{W}-L_{n t}^{A}-\sum_{r \in \Omega_{n}^{A}} \frac{1}{x^{\prime}}\right. \\
-\sum_{m \in \Psi_{n}^{A}} \frac{1}{x_{n m}}\left(\theta_{n t}^{A}-\theta_{m t}^{B}\right)=0, \forall n \in A
\end{gathered}
$$

$\theta_{1 t}^{A}=0, \delta_{1 w t}^{A}=0$, only for the reference area

$\left|\frac{1}{x_{n r}}\left(\theta_{n t}^{A}-\theta_{r t}^{A}\right)\right| \leq f_{n r}^{\max }, \forall(n, r) \in \Lambda^{A}$

$\left|\frac{1}{x_{n m}}\left(\theta_{n t}^{A}-\theta_{m t}^{B}\right)\right| \leq f_{n m}^{\max }, \forall(n, m) \in \Gamma^{A}$

$P_{i t}^{\mathrm{G}}+R_{i t}^{\mathrm{U}} \leq u_{i t} P_{i}^{\max }, \forall i \in A$

$P_{i t}^{\mathrm{G}}-R_{i t}^{\mathrm{D}} \geq u_{i t} P_{i}^{\mathrm{min}}, \forall i \in A$

$P_{i t}^{\mathrm{G}}-P_{i(t-1)}^{\mathrm{G}} \leq R U_{i}^{\mathrm{up}}, \forall i \in A$

$P_{i(t-1)}^{\mathrm{G}}-P_{i t}^{\mathrm{G}} \leq R D_{i}^{\text {down }}, \forall i \in A$

$0 \leq R_{i t}^{\mathrm{U}} \leq u_{i t} R_{i}^{\mathrm{U}, \max }, \forall i \in A$

$0 \leq R_{i t}^{\mathrm{D}} \leq u_{i t} R_{i}^{\mathrm{D}, \max }, \forall i \in A$

$0 \leq P_{j t}^{W} \leq P_{j t}^{\text {Offer }}, \forall j \in A$

$$
\begin{gathered}
\sum_{i \in \Phi_{n}^{\mathrm{G}, \mathrm{A}}}\left(r_{i w t}^{\mathrm{U}}-r_{i w t}^{\mathrm{D}}\right)+\sum_{j \in \Phi_{n}^{\mathrm{W}, \mathrm{A}}}\left(P_{j w t}^{W}-P_{j w t}^{\mathrm{spill}}-P_{j t}^{W}\right)+L_{n w t}^{\mathrm{shed}} \\
-\sum_{r \in \Omega_{n}^{A}} \frac{1}{x_{n r}}\left(\delta_{n w t}^{A}-\delta_{r w t}^{A}\right)-\sum_{m \in \Psi_{n}^{A}} \frac{1}{x_{n m}}\left(\delta_{n w t}^{A}-\delta_{m w t}^{B}\right) \\
=-\sum_{r \in \Omega_{n}^{A}} \frac{1}{x_{n r}}\left(\theta_{n t}^{A}-\theta_{r t}^{A}\right)-\sum_{m \in \Psi_{n}^{A}} \frac{1}{x_{n m}}\left(\theta_{n t}^{A}-\theta_{m t}^{B}\right), \\
\quad \forall n \in A, \forall w
\end{gathered}
$$




$$
\begin{aligned}
& \left|\frac{1}{x_{n r}}\left(\delta_{n w t}^{A}-\delta_{r w t}^{A}\right)\right| \leq f_{n r}^{\max }, \forall(n, r) \in \Lambda^{A}, \forall w \\
& \left|\frac{1}{x_{n m}}\left(\delta_{n w t}^{A}-\delta_{m w t}^{B}\right)\right| \leq f_{n m}^{\max }, \forall(n, m) \in \Gamma^{A}, \forall w \\
& 0 \leq r_{i w t}^{\mathrm{U}} \leq R_{i t}^{\mathrm{U}}, \forall w, \forall i \in A \\
& 0 \leq r_{i w t}^{\mathrm{D}} \leq R_{i t}^{\mathrm{D}}, \forall w, \forall i \in A \\
& 0 \leq P_{j w t}^{\mathrm{sill}} \leq P_{j w t}^{W}, \forall w, \forall j \in A \\
& 0 \leq L_{n w t}^{\text {shed }} \leq L_{n t}^{A}, \forall w, \forall n \in A \\
& u_{i t} \leq u_{i(t-1)}+y_{i t}, \forall i \in A \\
& \left.u_{i t} \in\{0,1\}, y_{i t} \in[0,1], \forall i \in A,\right\} \forall t, \forall A .
\end{aligned}
$$

The optimization variables of problem (1) are those in set $\Xi=$ $\left\{u_{i t}, y_{i t}, P_{i t}^{\mathrm{G}}, R_{i t}^{\mathrm{D}}, R_{i t}^{\mathrm{U}}, r_{i w t}^{\mathrm{U}}, r_{i w t}^{\mathrm{D}}, \forall i, \forall w ; P_{j t}^{W}, P_{j w t}^{\mathrm{spill}}, \forall j, \forall w\right.$; $\left.\theta_{n t}^{A}, \delta_{n w t}^{A}, L_{n w t}^{\text {shed }}, \forall n, \forall w\right\} \forall t, \forall A$.

The objective function (1a) to be minimized is the expected cost of the power system operation. The first row of (1a) includes the start-up costs, row 2 includes the cost of energy and reserve scheduling at the market stage, row 3 corresponds to the cost of redispatching (i.e., the actual deployment of upward/downward reserves by generating units) in real-time operation, and finally the last row of (1a) includes the cost of unserved energy in real-time operation.

Constraints (1b) are market balance equations. Constraints (1c) enforce $n=1$ to be the reference node. Constraints (1d) and (1e) enforce the transmission capacity limits of the internal lines and the tie-lines of each area, respectively. The set of constraints (1f) and (1g) enforces that the power plus the reserves scheduled in the market must be between the minimum power output and the capacity of the unit. Inter-temporal constraints (ramping up and ramping down limits) are enforced by (1h) and (1i). The upward/downward scheduled reserves are restricted by the upper limits $(1 \mathrm{j})$ and $(1 \mathrm{k})$ which represent market-fixed or physical limits. The scheduled wind power at the market stage is constrained by wind generating offer bounds (11). Constraints (1m) are power balance equations for border and interior buses in real-time operation. These constraints guarantee that any scenario realization is made feasible by redispatching generating units, incurring wind spillage, shedding loads and/or the assistance coming from neighboring areas. Constraints (1n) and (10), respectively, ensure that flows in all available interior and tie-lines under scenario $w$ are below their capacity limits. Constraints (1p) and (1q) state that the amount of upward/downward reserve that the available generating units can deploy is limited to the quantity established in the market. Constraints (1r) and (1s) set bound on the amount of wind power that can be spilled and on the amount of load that is involuntarily shed, respectively, under each scenario $w$. Constraints (1t) enforces the startup and shut down logic of production units.

\section{Area Decoupling Principle}

The area decoupling principle considered in this paper relies on adding fictitious nodes as explained in [11]; this principle is illustrated in Fig. 1. Consider Fig. 1(a) that shows the case of a single tie-line connecting the border bus $n$ of area A to the border bus $m$ of area B. In order to decouple per area this two-area system, one fictitious super node $z$, containing two nodes $z^{A}$ and $z^{B}$ assigned to areas $\mathrm{A}$ and $\mathrm{B}$, respectively, is included in the tie-line [see Fig. 1(b)]. Observe that these two nodes are connected to each other with negligible impedance.

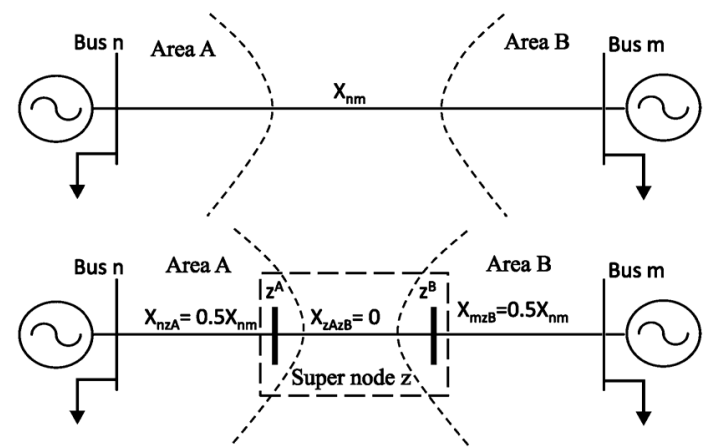

Fig. 1. Area decoupling principle.

Consistently with (1b) and (1m), the following load balance equations have to be enforced for the fictitious super node $z$ at the market stage (2a) and at real-time operation $(2 b)$ :

$$
\begin{aligned}
& \frac{2}{x_{n m}}\left(\theta_{n t}^{A}-\theta_{z^{A} t}^{A}\right)+\frac{2}{x_{n m}}\left(\theta_{m t}^{B}-\theta_{z^{B} t}^{B}\right)=0: \lambda_{n m, t}^{A, B} \\
& \left(\frac{2}{x_{n m}}\left(\delta_{n w t}^{A}-\delta_{z^{A} w t}^{A}\right)-\frac{2}{x_{n m}}\left(\theta_{n t}^{A}-\theta_{z^{A} t}^{A}\right)\right)+ \\
& \left(\frac{2}{x_{n m}}\left(\delta_{m w t}^{B}-\delta_{z^{B} w t}^{B}\right)-\frac{2}{x_{n m}}\left(\theta_{m t}^{B}-\theta_{z^{B} t}^{B}\right)\right)=0: \lambda_{n m, w t}^{A, B}
\end{aligned}
$$

where $\lambda_{n m, t}^{A, B}$ and $\lambda_{n m, w t}^{A, B}$, respectively, correspond to Lagrangian multiplier of equality constraints of (2a) and (2b) pertaining to fictitious super node $z$ (which has been added between border buses $n$ in area A and $m$ in area B) in period $t$. According to the above load balance equations, the centralized multi-area unit scheduling formulation (1) is modified as

$\underset{\Xi}{\operatorname{Minimize}}(1 \mathrm{a})$

subject to

$$
\begin{aligned}
& \left\{\sum_{i \in \Phi_{n}^{\mathrm{G}, \mathrm{A}}} P_{i t}^{\mathrm{G}}+\sum_{j \in \Phi_{n}^{\mathrm{W}, \mathrm{A}}} P_{j t}^{W}-L_{n t}^{A}\right. \\
& \quad-\sum_{r \in \Delta_{n}^{A}} \frac{1}{x_{n r}}\left(\theta_{n t}^{A}-\theta_{r t}^{A}\right)=0, \forall n \notin F^{A} \\
& \frac{2}{x_{n m}}\left(\theta_{n t}^{A}-\theta_{z^{A} t}^{A}\right)+\frac{2}{x_{n m}}\left(\theta_{m t}^{B}-\theta_{z^{B} t}^{B}\right) \\
& \quad=0: \lambda_{n m, t}^{A, B}, \forall n \in F^{A} \\
& \quad \sum_{i \in \Phi_{n}^{\mathrm{G}, \mathrm{A}}}\left(r_{i w t}^{\mathrm{U}}-r_{i w t}^{\mathrm{D}}\right)+\sum_{j \in \Phi_{n}^{\mathrm{w}, \mathrm{A}}}\left(P_{j w t}^{W}-P_{j w t}^{\mathrm{spill}}-P_{j t}^{W}\right) \\
& +L_{n w t}^{\mathrm{shed}}-\sum_{r \in \Delta_{n}^{A}} \frac{1}{x_{n r}}\left(\delta_{n w t}^{A}-\delta_{r w t}^{A}\right) \\
& +\sum_{r \in \Delta_{n}^{A}} \frac{1}{x_{n r}}\left(\theta_{n t}^{A}-\theta_{r t}^{A}\right)=0 \quad \forall n \notin F^{A}, \forall w \\
& \left(\frac{2}{x_{n m}}\left(\delta_{n w t}^{A}-\delta_{z^{A} w t}^{A}\right)-\frac{2}{x_{n m}}\left(\theta_{n t}^{A}-\theta_{z^{A} t}^{A}\right)\right) \\
& \quad+\left(\frac{2}{x_{n m}}\left(\delta_{m w t}^{B}-\delta_{z^{B} w t}^{B}\right)-\frac{2}{x_{n m}}\left(\theta_{m t}^{B}-\theta_{z^{B} t}^{B}\right)\right) \\
& \quad=0: \lambda_{n m, w t}^{A, B}, \forall n \in F^{A} \\
& (1 \mathrm{c})-(11),(1 \mathrm{n})-(1 \mathrm{u})\} \forall \mathrm{t}, \forall \mathrm{A} .
\end{aligned}
$$


Observe that the modified centralized model (3) is equivalent to the original centralized model (1).

\section{E. Decentralized Multi-Area Unit Scheduling Formulation}

In the decentralized formulation, particular attention should be paid to coupling constrains (3c) and (3e), since constraints (3b), (3d) and (3f) of the modified centralized problem (3) are easily decoupled by area.

The augmented Lagrange relaxation (ALR) algorithm described in [9] and [17] is employed in this paper to decompose problem (3) per area by relaxing all the coupling constraints. The required dual problem is formulated as (see [9] or [17]):

Maximize $\phi(\lambda)$

$$
\begin{aligned}
\phi(\lambda)= & \underset{\Xi}{\operatorname{Minimize}}(1 \mathrm{a})+\sum_{A} \sum_{(n, m) \in \Gamma^{A}} \sum_{t}^{N_{T}} \\
\times & \left\{\lambda_{n m, t}^{A, B}\left[\frac{2}{x_{n m}}\left(\theta_{n t}^{A}-\theta_{z^{A} t}^{A}\right)+\frac{2}{x_{n m}}\left(\theta_{m t}^{B}-\theta_{z^{B} t}^{B}\right)\right]\right. \\
+ & \sum_{w=1}^{N_{W}} \lambda_{n m, w t}^{A, B} \\
\times & {\left[\left(\frac{2}{x_{n m}}\left(\delta_{n w t}^{A}-\delta_{z^{A} w t}^{A}\right)-\frac{2}{x_{n m}}\left(\theta_{n t}^{A}-\theta_{z^{A} t}^{A}\right)\right)\right.} \\
& \left.+\left(\frac{2}{x_{n m}}\left(\delta_{m w t}^{B}-\delta_{z^{B} w t}^{B}\right)-\frac{2}{x_{n m}}\left(\theta_{m t}^{B}-\theta_{z^{B} t}^{B}\right)\right)\right] \\
+ & \frac{\eta}{2}\left\|\frac{2}{x_{n m}}\left(\theta_{n t}^{A}-\theta_{z^{A} t}^{A}\right)+\frac{2}{x_{n m}}\left(\theta_{m t}^{B}-\theta_{z^{B} t}^{B}\right)\right\|^{2}+\sum_{w=1}^{N_{W}} \frac{\xi}{2} \\
\times & \|\left(\frac{2}{x_{n m}}\left(\delta_{n w t}^{A}-\delta_{z^{A} w t}^{A}\right)-\frac{2}{x_{n m}}\left(\theta_{n t}^{A}-\theta_{z^{A} t}^{A}\right)\right) \\
& \left.+\left(\frac{2}{x_{n m}}\left(\delta_{m w t}^{B}-\delta_{z^{B} w t}^{B}\right)-\frac{2}{x_{n m}}\left(\theta_{m t}^{B}-\theta_{z^{B} t}^{B}\right)\right) \|^{2}\right\}
\end{aligned}
$$

subject to

$$
\text { (3b), (3d), (3f), } \forall \mathrm{t}, \forall \mathrm{A} \text {. }
$$

We note that the quadratic terms defined above have a favorable and significantly effect on convergence. However, these quadratic terms are not separable and the problem cannot be solved per area. Nevertheless, as discussed in [2], the quadratic terms of the augmented Lagrangian can be linearized by fixing a minimum number of variables to the values of the previous iteration to achieve separability. This approach, known as auxiliary problem principle (APP), was proposed in [18]. Another coordination method, known as alternative direction method (ADM), that directly fixes in the augmented Lagrangian the minimum number of variables to the values of the previous iteration to achieve separability is reported in [19]. To decompose the original problem, we select the APP technique as it generally exhibits a better convergence behavior than the ADM technique in the case of non-convexity.

Regarding the APP technique, it is relevant to note that the so-called core functions $\mathrm{K}$, as stated in [18], are considered quadratic and additive with respect to the areas' border variables. For further discussion on this issue the interested reader is referred to [18].
By applying the APP technique, problem (4) splits into $|A|$ (the number of areas) independent problems, one per area. Considering area decomposition, the $\nu^{\text {th }}$ iteration of this algorithm under certain conditions can be written as follows:

$$
\begin{aligned}
& \left\{\phi\left(\lambda^{A}\right)^{\nu+1}\right. \\
& =\underset{\Xi_{A}}{\operatorname{Minimize}}(1 \mathrm{a}) \\
& +\sum_{(n, m) \in \Gamma^{A}} \frac{2\left(\lambda_{n m, t}^{A, B}\right)^{\nu}}{x_{n m}}\left[\left(\theta_{n t}^{A}-\theta_{z^{A} t}^{A}\right)\right] \\
& +\sum_{(n, m) \in \Gamma^{A}} \frac{2 \gamma}{x_{n m}^{2}}\left\|\left(\theta_{n t}^{A}-\theta_{z^{A} t}^{A}\right)-\left(\left(\widehat{\theta}_{n t}^{A}\right)^{\nu}-\left(\widehat{\theta}_{z^{A} t}^{A}\right)^{\nu}\right)\right\|^{2} \\
& +\sum_{(n, m) \in \Gamma^{A}} \frac{4 \eta}{x_{n m}^{2}} \\
& \times\left[\left(\theta_{n t}^{A}-\theta_{z^{A} t}^{A}\right) \times\left(\left(\left(\widehat{\theta}_{n t}^{A}\right)^{\nu}-\left(\widehat{\theta}_{z^{A} t}^{A}\right)^{\nu}\right)\right.\right. \\
& \left.\left.+\left(\left(\widehat{\theta}_{m t}^{B}\right)^{\nu}-\left(\widehat{\theta}_{z^{B} t}^{B}\right)^{\nu}\right)\right)\right] \\
& +\sum_{w=1}^{N_{W}} \sum_{(n, m) \in \Gamma^{A}} \frac{2\left(\lambda_{n m, s t}^{A, B}\right)^{\nu}}{x_{n m}} \\
& \times\left[\left(\delta_{n w t}^{A}-\delta_{z^{A} w t}^{A}\right)-\left(\theta_{n t}^{A}-\theta_{z^{A} t}^{A}\right)\right] \\
& +\sum_{w=1}^{N_{W}} \sum_{(n, m) \in \Gamma^{A}} \frac{2 \zeta}{x_{n m}^{2}} \\
& \times \|\left[\left(\delta_{n w t}^{A}-\delta_{z^{A} s t}^{A}\right)-\left(\theta_{n t}^{A}-\theta_{z^{A} t}^{A}\right)\right] \\
& -\left[\left(\left(\widehat{\delta}_{n w t}^{A}\right)^{\nu}-\left(\widehat{\delta}_{z^{A} w t}^{A}\right)^{\nu}\right)-\left(\left(\widehat{\theta}_{n t}^{A}\right)^{\nu}-\left(\widehat{\theta}_{z^{A} t}^{A}\right)^{\nu}\right)\right] \|^{2} \\
& +\sum_{w=1}^{N_{W}} \sum_{(n, m) \in \Gamma^{A}} \frac{4 \xi}{x_{n m}^{2}}\left[\left(\delta_{n w t}^{A}-\delta_{z^{A} s t}^{A}\right)-\left(\theta_{n t}^{A}-\theta_{z^{A} t}^{A}\right)\right] \\
& \times\left[\left(\left(\widehat{\delta}_{n w t}^{A}\right)^{\nu}-\left(\widehat{\delta}_{z^{A} w t}^{A}\right)^{\nu}\right)-\left(\left(\widehat{\theta}_{n t}^{A}\right)^{\nu}-\left(\widehat{\theta}_{z^{A} t}^{A}\right)^{\nu}\right)\right. \\
& \left.+\left(\left(\widehat{\delta}_{m w t}^{B}\right)^{\nu}-\left(\widehat{\delta}_{z^{B} w t}^{B}\right)^{\nu}\right)-\left(\left(\widehat{\theta}_{m t}^{B}\right)^{\nu}-\left(\widehat{\theta}_{z^{B} t}^{B}\right)^{\nu}\right)\right]
\end{aligned}
$$

subject to

$$
(3 \mathrm{~b}),(3 \mathrm{~d}),(3 \mathrm{f}), \quad\} \forall \mathrm{t}, \forall \mathrm{A}
$$

$$
\begin{aligned}
& \left(\lambda_{n m, t}^{A, B}\right)^{\nu+1} \\
& =\left(\lambda_{n m, t}^{A, B}\right)^{\nu} \\
& \quad+\alpha\left[\left(\left(\widehat{\theta}_{n t}^{A}\right)^{\nu}-\left(\widehat{\theta}_{z^{A} t}^{A}\right)^{\nu}\right)+\left(\left(\widehat{\theta}_{m t}^{B}\right)^{\nu}-\left(\widehat{\theta}_{z^{B} t}^{B}\right)^{\nu}\right)\right] \\
& \quad(5 \mathrm{c}) \\
& \left(\lambda_{n m, w t}^{A, B}\right)^{\nu+1} \\
& =\left(\lambda_{n m, s t}^{A, B}\right)^{\nu} \\
& \quad+\beta\left[\left(\left(\widehat{\delta}_{n w t}^{A}\right)^{\nu}-\left(\widehat{\delta}_{z^{A} w t}^{A}\right)^{\nu}\right)-\left(\left(\widehat{\theta}_{n t}^{A}\right)^{\nu}-\left(\widehat{\theta}_{z^{A} t}^{A}\right)^{\nu}\right)\right. \\
& \left.\quad+\left(\left(\widehat{\delta}_{m w t}^{B}\right)^{\nu}-\left(\widehat{\delta}_{z^{B} w t}^{B}\right)^{\nu}\right)-\left(\left(\widehat{\theta}_{m t}^{B}\right)^{\nu}-\left(\widehat{\theta}_{z^{B} t}^{B}\right)^{\nu}\right)\right] .
\end{aligned}
$$


The optimization variables of problem (5) are those in set $\Xi_{A}=\left\{u_{i t}, y_{i t}, P_{i t}^{\mathrm{G}}, R_{i t}^{\mathrm{D}}, R_{i t}^{\mathrm{U}}, r_{i w t}^{\mathrm{U}}, r_{i w t}^{\mathrm{D}}, \forall i, \forall w ; P_{j t}^{W}, P_{j w t}^{\mathrm{spill}}\right.$, $\left.\forall j, \forall w ; \theta_{n t}^{A}, \delta_{n w t}^{A}, L_{n w t}^{\text {shed }}, \forall n, \forall w\right\} \forall t$.

Observe that problem (4) has been effectively decomposed into smaller problems (5) for each area. Such decomposed problems are iteratively solved until the optimal solution is achieved. A subgradient algorithm is used to solve the dual problem (i.e., for updating the Lagrange multipliers) through (5c) and (5d).

\section{F. On Convergence, Information Interchange, and Implementation}

The proposed decentralized algorithm relies on the APP that allows us to cope with the non-separable quadratic terms introduced by augmented Lagrange relaxation. The decoupled solution of the centralized problem (1) can be achieved under certain conditions by solving the areas' problems (5) iteratively.

At the end of each iteration $\nu$, each area A interchanges with neighboring areas the state variables (i.e., voltage phase angles of border buses under market and real-time operation) of border and fictitious buses, $\left(\widehat{\theta}_{n t}^{A}\right)^{\nu},\left(\widehat{\theta}_{z^{A} t}^{A}\right)^{\nu},\left(\widehat{\delta}_{n w t}^{A}\right)^{\nu}$, and $\left(\widehat{\delta}_{z^{A} w t}^{A}\right)^{\nu}$. Additionally, each area A updates the Lagrange multipliers of the coupling constraints using its own information and the information received from neighboring areas by $(5 c)$ and $(5 d)$. The algorithm converges if the $L^{2}$-norm of the coupling constraints evaluated at $\left(\widehat{\Xi}_{A}, \forall A\right)$ is satisfied within a tolerance $\epsilon$. Moreover, observe that the exchanged information is moderate and no central coordinator is required.

Note that although the convergence of the APP technique is only guaranteed in the case of a convex problem, this algorithm can still be used in practical non-convex problems in the heuristic hope of achieving convergence. In case of a nonconvex problem, if convergence is indeed achieved, the optimality conditions are satisfied locally [18].

Our experience shows that the convergence of this algorithm is generally sensitive to the values of the weighting parameter, and not to the network topology. However, for the considered case study we have found empirically that convergence is efficiently attained with the choice of $\gamma \geq 2 \eta=2 \alpha$ and $\zeta \geq 2 \xi=$ $2 \beta$. Nonetheless, note that these parameter choices should be tuned up for each system under consideration.

A description of the proposed algorithm to clear the multi-regional electricity markets in a decentralized procedure is shown in Fig. 2. It is important to note that a sequential solution of the area subproblems is required. That is, the solution of area 2 has to be carried out after solving the subproblem of area 1 , and the problem of area 3 has to be solved after solving the problem of area 2 and so forth. This is a consequence of considering just one fictitious super node per tie-line. Two fictitious nodes per line [11] can be used to achieve a parallel implementation.

Observe that the selection of reference voltage angles in this sequential solution scheme deserves attention. One bus (e.g., $n=1$ ) in area A is selected as reference bus. Once the subproblem of area $\mathrm{A}$ is solved in iteration $\nu$, the value of voltage angle of fictitious bus $z^{A}$ is fixed to voltage angle value of node $z^{B}$ of area B; i.e., $\theta_{z^{B} t}^{B}=\left(\widehat{\theta}_{z^{A} t}^{A}\right)^{\nu}$ and $\delta_{z^{B} w t}^{B}=\left(\widehat{\delta}_{z^{A} w t}^{A}\right)^{\nu}$.

Additionally, we have found experimentally that it is computationally advantageous that all areas ignore the coordination mechanism in the first iteration (i.e., each areas has its own slack bus in the first iteration) and focus on minimizing their specific objectives, and right after the first iteration the coordination

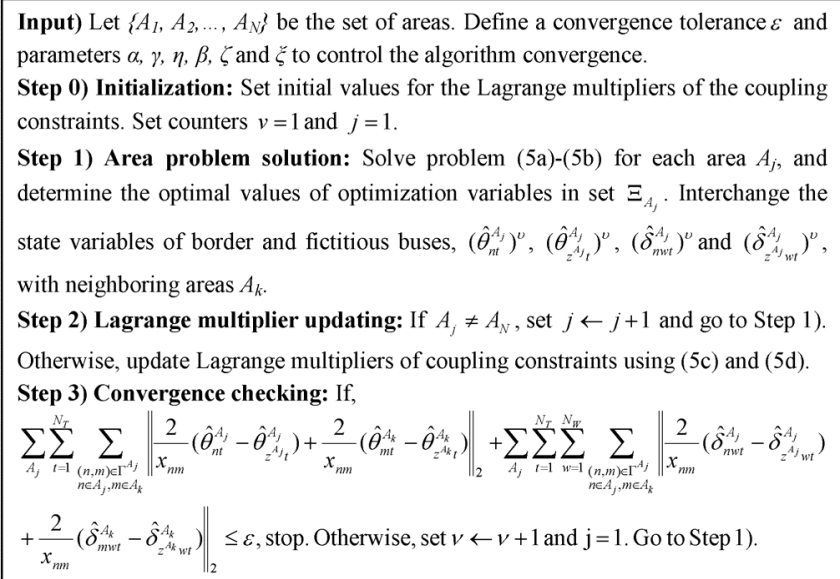

Fig. 2. Decentralized market-clearing algorithm procedure (sequential form).

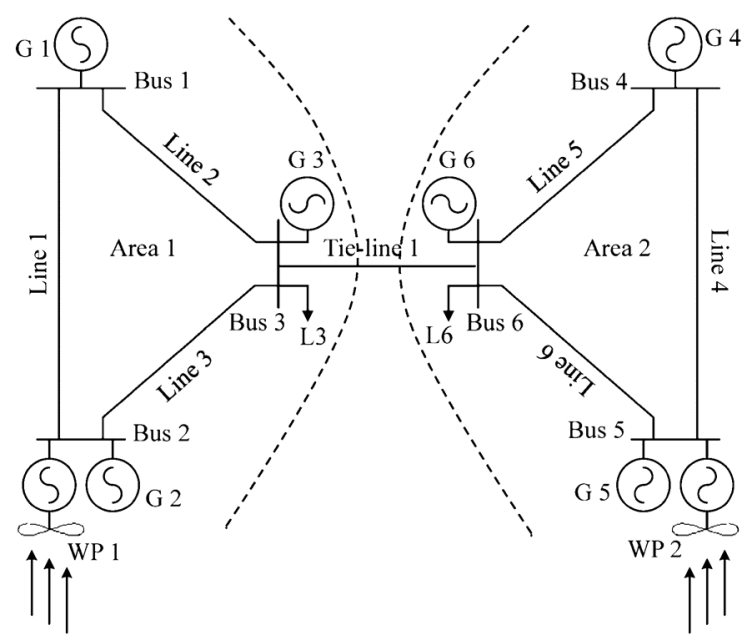

Fig. 3. Six-bus interconnected test system.

mechanism is enforced. This way, the convergence behavior of the algorithm is enhanced, since it avoids initial fighting among areas to impose their respective positions with respect to the positions of other areas.

\section{IlluUSTRATIVE EXAMPLE AND CASE StUdy}

Results from two test cases are presented in this section: a six-bus interconnected test system and the three-area IEEE-RTS. The model has been implemented on a Sun Fire X4600M2 with 8 Quad-Core processors, $2.9 \mathrm{GHz}$ and $256 \mathrm{~GB}$ of RAM, using CPLEX 12.1.0 under GAMS [20].

\section{A. Six-Bus Interconnected Test System}

The considered example is depicted in Fig. 3. Two identical systems are interconnected through one tie-line.

Line reactances (internals and tie-line) are all 0.13 p.u. on a base of 100 MVA. The capacities of all internal lines are equal to $100 \mathrm{MW}$, and the capacity of the tie-line is set to $50 \mathrm{MW}$.

The generating unit data are given in Table I. Observe that the costs of generators of area 2 for energy and reserve are twice those of generators in area 1 . This way, we force power imports on area 2. For the sake of simplicity, it is assumed that the energy and reserve offers of the units in both areas remain unchanged throughout the scheduling horizon. The ramping capabilities of 
TABLE I

CONVENTIONAL GENERATING UNITS DATA

\begin{tabular}{c|cccccc}
\hline Generator $i$ & 1 & 2 & 3 & 4 & 5 & 6 \\
\hline$P_{i}^{\max }(\mathrm{MW})$ & 100 & 100 & 50 & 100 & 100 & $\mathbf{5 0}$ \\
\hline$P_{i}^{\min }(\mathrm{MW})$ & 10 & 10 & 10 & 10 & 10 & 10 \\
\hline$C_{i t}^{\mathrm{SU}}(\$)$ & 100 & 100 & 100 & 200 & 200 & 200 \\
\hline$a_{i}(\$ / \mathrm{h})$ & 100 & 100 & 100 & 200 & 200 & 200 \\
\hline$b_{i}(\$ / \mathrm{MWh})$ & 30 & 40 & 20 & 60 & 80 & 40 \\
\hline$c_{i}\left(\$ / \mathrm{MW}^{2} \mathrm{~h}\right)$ & 0.3 & 0.8 & 0.2 & 0.6 & 1.6 & 0.4 \\
\hline$q_{i t}^{\mathrm{U}}($ \$/MW\&h $)$ & 3 & 4 & 2 & 6 & 8 & 4 \\
\hline$q_{i t}^{\mathrm{D}}(\$ / \mathrm{MW} \& \mathrm{~h})$ & 3 & 4 & 2 & 6 & 8 & 4 \\
\hline
\end{tabular}

TABLE II

HOURLY LOAD DATA

\begin{tabular}{ccccc}
\hline Period t & 1 & 2 & 3 & 4 \\
\hline L3 (MW) & 50 & 110 & 140 & 80 \\
\hline L6 (MW) & 50 & 110 & 140 & 80 \\
\hline
\end{tabular}

TABLE III

WIND POWER SCENARIOS (IN MW)

\begin{tabular}{cccccc}
\hline \multirow{2}{*}{ Period t } & \multicolumn{5}{c}{ Wind Scenarios } \\
\cline { 2 - 6 } & $(\mathrm{M}, \mathrm{M})$ & $(\mathrm{H}, \mathrm{H})$ & $(\mathrm{L}, \mathrm{L})$ & $(\mathrm{H}, \mathrm{L})$ & $(\mathrm{H}, \mathrm{M})$ \\
\hline 1 & $(6,6)$ & $(9,9)$ & $(2,2)$ & $(9,2)$ & $(9,6)$ \\
\hline 2 & $(20,20)$ & $(30,30)$ & $(13,13)$ & $(30,13)$ & $(30,20)$ \\
\hline 3 & $(35,35)$ & $(50,50)$ & $(25,25)$ & $(50,25)$ & $(50,35)$ \\
\hline 4 & $(8,8)$ & $(12,12)$ & $(6,6)$ & $(12,6)$ & $(12,8)$ \\
\hline
\end{tabular}

generating units are the largest possible, i.e., $P_{i}^{\max } \mathrm{MW} / \mathrm{h}$. Furthermore, the six generators are assumed to be offline at the beginning of the study horizon $(t=0)$.

The generation mix includes two wind power plants (WP) located at bus 2 and bus 5 as indicated in Fig. 3. The forecast production of both wind generators for market scheduling is 35 MW.

Two demands with the hourly load profile detailed in Table II are located at buses 3 and 6 , respectively. The value of unserved load is $1000 \$ / \mathrm{MWh}$. The model is tested over a 4-h scheduling horizon.

The data provided so far for this small-scale six-bus test system defines the base case.

We examine below the impact of wind production uncertainty on the generating units and reserve scheduling of this base case. To this end, we consider the five wind production scenarios listed in Table III, identified as "both areas medium (M,M)", "both areas high $(\mathrm{H}, \mathrm{H})$ ", "both areas low $(\mathrm{L}, \mathrm{L})$ ", " area 1 high and area 2 low $(\mathrm{H}, \mathrm{L})$ " and "area 1 high and area 2 medium $(\mathrm{H}, \mathrm{M})$ ", with probabilities of occurrence, $0.6,0.1,0.1,0.1$ and 0.1 , respectively.

This base case is solved in centralized and decentralized fashions. Results are identical. These results are provided in Fig. 4 and Table IV. Black dots in Fig. 4 indicate the units that are committed. From Table IV, observe that the CPU time required by the centralized procedure is smaller than that required by the decentralized procedure. However, recall that the decomposition is not oriented to improve computational efficiency but to preserve scheduling independence. In fact, the centralized procedure is used here to validate the results obtained by the de-
Centralized Decentralized

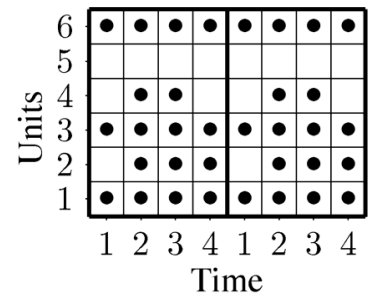

Fig. 4. Units status in both centralized and decentralized approaches.

TABLE IV

COMPARISON OF CENTRALIZED AND DECENTRALIZED Models

\begin{tabular}{cccc}
\hline & $\begin{array}{c}\text { Total Cost } \\
(\$)\end{array}$ & $\begin{array}{c}\text { CPU Time } \\
\text { (second) }\end{array}$ & $\begin{array}{c}\text { Iteration } \\
\text { numbers }\end{array}$ \\
\hline Centralized Model & 32272 & 0.25 & 1 \\
\hline Decentralized Model & 32271 & 8 & 21 \\
\hline
\end{tabular}

TABLE V

MARKet OUTCOMES-SiX-Bus System. Powers IN MW

\begin{tabular}{ccccccc}
\hline & & & \multicolumn{4}{c}{ Period $t$} \\
\cline { 3 - 7 } & & Unit & 1 & 2 & 3 & 4 \\
\hline & & $\mathrm{G} 1$ & 27.3 & 63.5 & 78.0 & 50.6 \\
& $P_{i t}^{\mathrm{G}}$ & $\mathrm{G} 2$ & 0 & 17.6 & 21.4 & 13.5 \\
Area 1 & & $\mathrm{G} 3$ & $\mathbf{5 0 . 0}$ & $\mathbf{5 0 . 0}$ & $\mathbf{5 0 . 0}$ & $\mathbf{5 0 . 0}$ \\
& $P_{j t}^{W}$ & $\mathrm{~W} 1$ & 1.8 & 16.5 & 30.6 & 3.5 \\
\cline { 2 - 7 } & \multirow{2}{*}{$R_{i t}^{\mathrm{U}}$} & $\mathrm{G} 1$ & 8.7 & 15.4 & 14.4 & 7.0 \\
& & $\mathrm{G} 2$ & 0 & 0.5 & 1.3 & 0 \\
\cline { 2 - 7 } & \multirow{2}{*}{$R_{i t}^{\mathrm{D}}$} & $\mathrm{G} 1$ & 5.4 & 14.5 & 23.1 & 5.0 \\
& & $\mathrm{G} 2$ & 0 & 0.5 & 1.2 & 0 \\
\hline \multirow{4}{*}{ Area 2 } & $P_{i t}^{\mathrm{G}}$ & $\mathrm{G} 4$ & 0 & 10.0 & 15.0 & 0 \\
& $P_{j t}^{W}$ & $\mathrm{G} 6$ & 10.0 & 35.5 & 50.0 & 27.0 \\
\cline { 2 - 7 } & $R_{i t}^{\mathrm{D}}$ & $\mathrm{G} 2$ & 10.8 & 26.9 & 35.0 & 15.5 \\
\cline { 2 - 7 } & $R_{i t}^{\mathrm{D}}$ & $\mathrm{G} 6$ & 0 & 1.5 & 0 & 0 \\
\hline
\end{tabular}

centralized procedure. In what follows only the results of the decentralized model are analyzed.

The market outcomes related to units scheduling and the reserve allocation are presented in Table V. It is relevant to point out that no load shedding and no wind spillage occur during the entire scheduling horizon and under any scenario, and that the unexpected events in a given area are covered only by deploying upward/downward reserve and assistance from the other area.

Figs. 5 and 6 show the evolution of the marginal price of the tie-line, $\lambda_{36,3}^{1,2}$, and tie-line flow (from both directions, i.e., from bus 3 to fictitious bus 7 in area 1 and from fictitious bus 8 to bus 6 in area 2) at the peak time period (period 3) as a function of the iteration number, respectively. It is observed that the convergence is smoothly attained after 21 iterations within a tolerance $\epsilon=10^{-3}$.

Note that during the first iteration both areas ignore the coordination mechanism and both areas seek to import the maximum possible power $(40 \mathrm{MW})$ from the neighbor area. This is so because the marginal price at the tie-line $\left(\lambda_{36}^{1,2}\right)$ is zero in the first iteration (flat start). Once the coordination mechanism is enforced and the marginal price at the tie-line is updated (increasing as shown in Fig. 5), area 1 increases its productions while simultaneously decreasing its import from area 2 . 


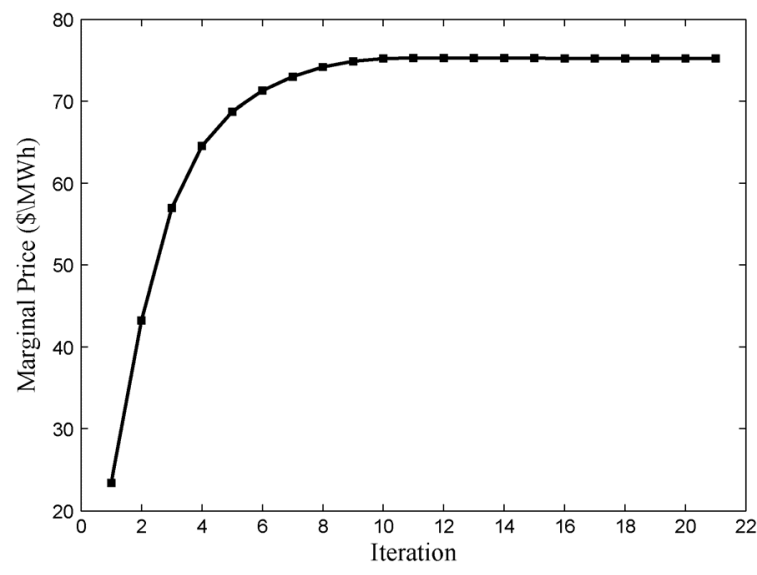

Fig. 5. Evolution of marginal prices of tie-line at peak time period $\lambda_{36,3}^{1,2}$.

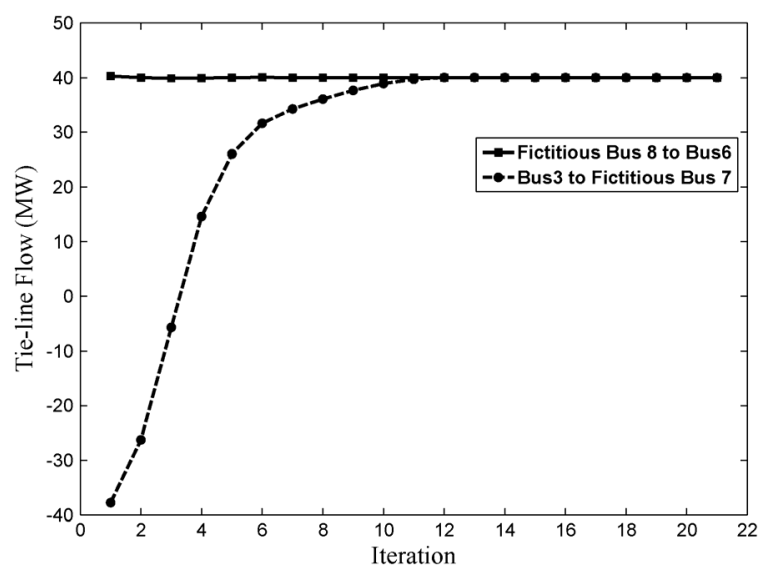

Fig. 6. Evolution of tie-line flow at peak time period.

We note that the production in area 2 does not change significantly, as area 2 still imports a similar level of power from area 1. This is so because it is cheaper for area 2 to import power from area 1 , since the value of the tie-line marginal cost is lower than the marginal cost of the most expensive unit in area 2. Starting at iteration 4 , area 1 no longer import power from area 2, since the marginal price at the tie-line is higher than the marginal cost of the most expensive unit in area 1. Thus, from this iteration on, area 1 changes from importer to exporter, while area 2 still keeps importing the maximum possible power from area 1 (see Fig. 6).

The benefits of the interconnection are illustrated in Table VI. This table compares the market-clearing results, including total generation, total reserve, and total cost of each area, for the cases with (tie-line capacity set to $50 \mathrm{MW}$ ) and without (tie-line capacity set to $0 \mathrm{MW}$ ) interconnection. It can be observed from Table VI that the total cost decreases if the system is interconnected. Note also that the power and reserve requirements of area 2 (costly area) are partly covered by inexpensive generating units in area 1. Both areas benefit from inter-regional trading: area 2 by buying cheap and area 1 by selling more.

It is relevant to analyze the impact of wind power uncertainty and tie-line capacity on the problem outcomes. For this purpose, the base case is next solved for a large set of conditions characterized by different wind power uncertainty and tie-line capacities. The wind power uncertainty level is defined by using different sets of scenarios having the same average hourly value
TABLE VI

COMPARISON OF MARKET-CLEARING RESULTS: INTERCONNECTED VERSUS ISOLATED OPERATION

\begin{tabular}{lcccc}
\hline & & Area 1 & Area 2 & System wide \\
\hline \multirow{2}{*}{$\begin{array}{l}\text { Interconnected } \\
\text { Operation }\end{array}$} & $\begin{array}{c}\text { Total Generation } \\
(\mathrm{MWh})\end{array}$ & 531.7 & 228.3 & 760 \\
\cline { 2 - 5 } & $\begin{array}{c}\text { Total Reserve } \\
(\mathrm{MW})\end{array}$ & 97.3 & 12.7 & 110 \\
\cline { 2 - 5 } & $\begin{array}{c}\text { Total Cost } \\
(\$)\end{array}$ & 21323 & 10949 & 32272 \\
\hline \multirow{3}{*}{$\begin{array}{l}\text { Tsolated } \\
\text { Operation }\end{array}$} & $\begin{array}{c}\text { Total Generation } \\
(\mathrm{MWh})\end{array}$ & 380 & 380 & 760 \\
\cline { 2 - 5 }$(\mathrm{MW})$ & 55 & 55 & 110 \\
\hline & $\begin{array}{c}\text { Total Cost } \\
(\$)\end{array}$ & 11972 & 24740 & 36712 \\
\hline
\end{tabular}

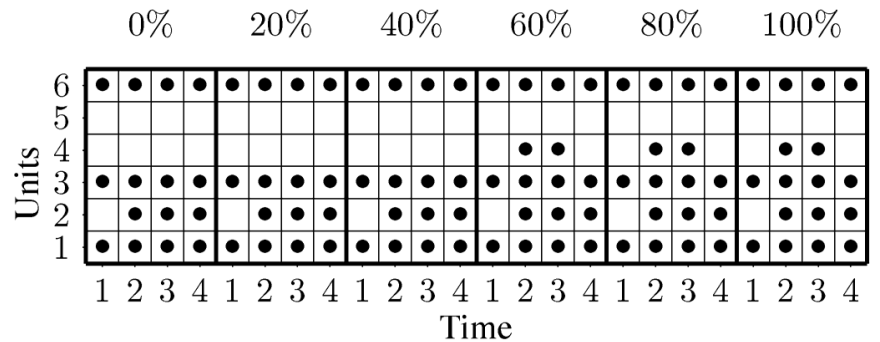

Fig. 7. Generating units status versus wind power uncertainty.

(i.e., the average scenario is the same for all sets), but different hourly variances $(x \%)$. To do so, the high and low scenarios are defined to be equal to the wind medium scenario multiplied by $(1+x / 100)$ and $(1-x / 100)$, respectively.

Fig. 7 shows how the $0 / 1$ unit commitment decisions change with the wind power uncertainty level; black dots indicate the units that are committed. Observe that more units are committed as the level of wind power uncertainty increases (check unit G4 at uncertainty level $60 \%$ ). Additionally, an increasing trend of the reserve needed can be observed in Fig. 8 as the wind power uncertainty increases. Quantitatively, it can be inferred from this figure that most of the required reserve is allocated to area 1 , which is less costly.

Fig. 9 illustrates the evolution of unit schedules (on/off) obtained for different tie-line capacities ranging from $0 \mathrm{MW}$ to 100 $\mathrm{MW}$ at 25-MW increments. It is clear that increasing the tie-line capacity can have significant effect on the unit scheduling, as several expensive units will not be scheduled at some time periods. Fig. 10 depicts the variation of the share of the reserve allocated for increasing values of the capacity of the tie-line (0 to $100 \mathrm{MW}$ ). By increasing the tie-line capacity, the congestion is relieved and cross border trade (energy and reserve sharing) rises to more desirable values. It can be observed in Fig. 10 that the total share of the reserve allocated to area 1 (inexpensive area) increases and the share of area 2 (costly area) decreases monotonically as the tie-line capacity increases.

\section{B. Three-Area IEEE-RTS}

Results from a large case study based on the three-area version of IEEE Reliability Test System-1996 [21] are discussed in this subsection. Note that the connections among the three areas of the IEEE 3-area RTS are triangular. 


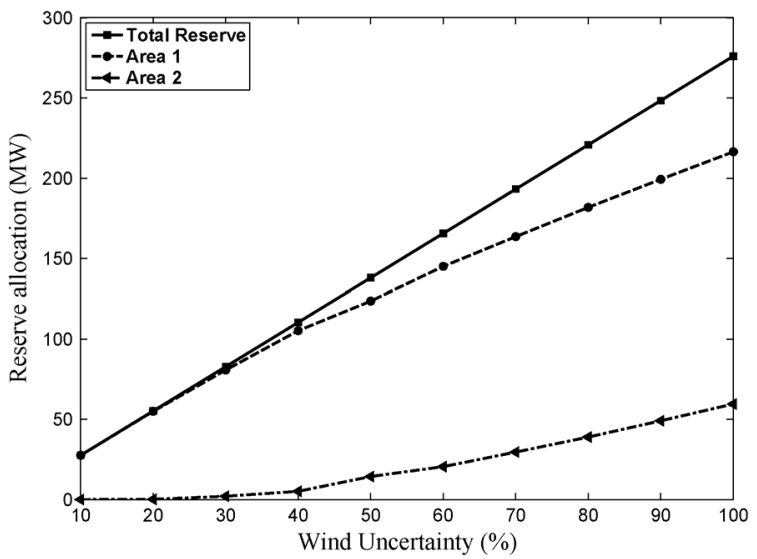

Fig. 8. Total share of reserve versus wind power uncertainty.

0MW 25MW 50MW $75 \mathrm{MW} \quad 100 \mathrm{MW}$

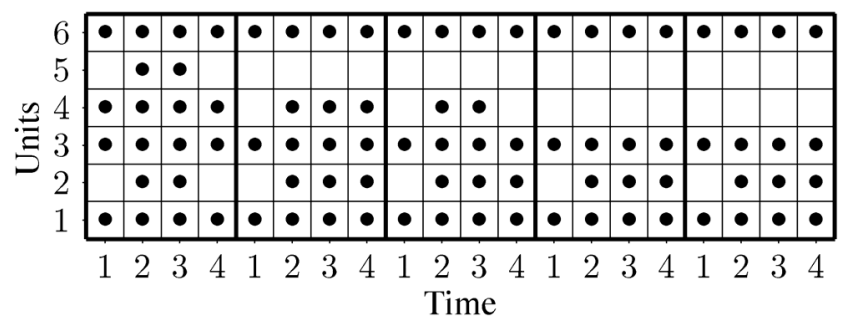

Fig. 9. Generating units status versus tie-line capacity.

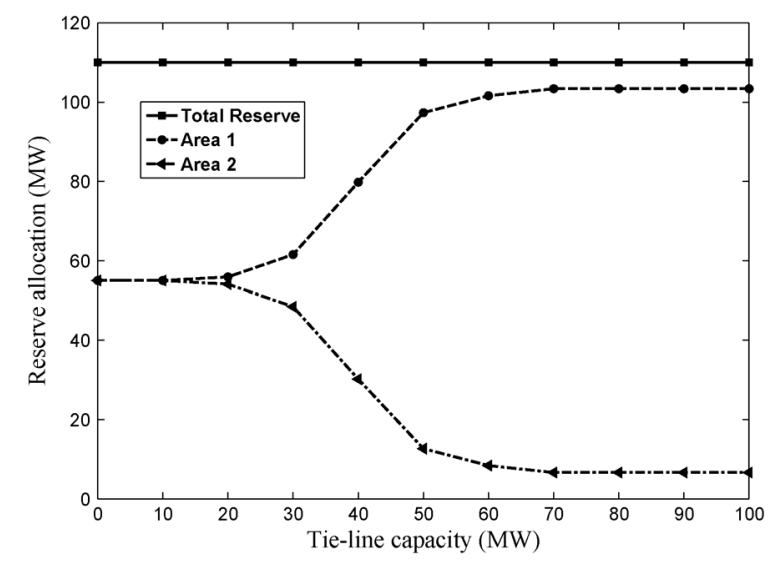

Fig. 10. Total share of reserve versus tie-line capacity.

TABLE VII

Fuel Cost DATA

\begin{tabular}{cccccc}
\hline Fuel Type & FO6 & FO2 & Coal & Nuclear & Hydro \\
\hline Cost $(\$ / M B T U)$ & 2.3 & 3.0 & 1.2 & 0.6 & 0.0 \\
\hline
\end{tabular}

The offer submitted by generating units correspond to their marginal costs of energy production, which are derived from the heat rate data provided in [21] (fitted by quadratic functions) and the fuel cost data listed in Table VII. Besides, each unit offers the maximum possible upward/downward reserve at a price equal to $10 \%$ of the coefficient $b$ of its cost function. To force crossborder trading, the cost of units in areas 2 for both energy and reserve are assumed to be twice those of units in areas 1 and 3.
TABLE VIII

COMPARISON OF CENTRALIZED AND DECENTRALIZED MODELS. THREE-AREA IEEE-RTS

\begin{tabular}{lccc}
\hline & $\begin{array}{c}\text { Total Cost } \\
(\$)\end{array}$ & $\begin{array}{c}\text { CPU Time } \\
\text { (second) }\end{array}$ & $\begin{array}{c}\text { Iteration } \\
\text { numbers }\end{array}$ \\
\hline Centralized Model & 1504950 & 106 & 1 \\
\hline Decentralized Model & 1509853 & 604 & 46 \\
\hline
\end{tabular}

The original data is modified to include two wind power plants of $300 \mathrm{MW}$ and $100 \mathrm{MW}$ at buses 107 in area 1 and 207 in area 2 , respectively.

For modeling the most plausible realizations of wind power throughout the scheduling horizon, and in order to make this case study sufficiently realistic, an initial set of 276 equally probable wind generation scenarios are considered using publicly available wind power data for year 2012 from two locations in West and East Denmark [22]. Thus, to achieve tractability, these scenarios are reduced to 10 by applying the scenario reduction technique proposed in [23]. Note that the corresponding scenarios are scaled down according to the wind power plant capacities considered in this case study.

The load profile corresponds to a winter Thursday (week 45) with a peak load of $2850 \mathrm{MW}$ for each area (8550 MW for the 3 -area system). The value of lost load for all demands is assumed to be $1000 \$ / M W h$. The scheduling time horizon considered is one day on a 2 -h basis, i.e., we bundle the hourly scheduling horizon by $2, d_{t}=2, \forall t$. This way we can solve the proposed model in a reasonable time using an ordinary computer.

Moreover, to decrease the computational burden, the nodes connected through lines that are not likely to suffer congestion are gathered into a single node without altering the results of the study. Therefore, we reduce the number of nodes in each area to eight, merging nodes 1 to 13 , nodes 17 to 20 and nodes 21 to 22 into single nodes. By trial and error, we found that for parameters $\alpha=\eta=0.4 \gamma$ and $\beta=\xi=0.4 \zeta$ the convergence is robust in this case study. The value of tolerance is set to $\epsilon=10^{-3}$.

We compare in Table VIII the results of the centralized procedure (total cost and the CPU time) with those pertaining of decentralized procedure. From the comparison, it can be concluded that the total cost of these two procedures are close enough. However, the CPU time of the decentralized solution is higher than that of the centralized one.

Nevertheless, recall that the purpose of decentralized model is not improving the computing speed, but preserving the area scheduling independence. It is stressed again that what our paper does is providing an approach to operate a system with significant interconnections and wind integration in a decentralized manner, and fully preserving the advantage of the interconnections.

\section{CONCLUSION}

This paper provides a methodology to determine unit scheduling and reserve allocation in an interconnected multi-area power system under wind power uncertainty. The theoretical properties of the proposed model and extensive numerical simulations allow concluding the following:

1) The proposed decentralized technique is accurate, as the final results are equal to those obtained by a centralized procedure using the whole information available in all areas. 
2) The units schedules and the geographical allocation of reserve in a particular area are dependent on both the wind power uncertainty level and the tie-line capacities between areas.

3) Time steps of more than 1 hour may be needed to solve the problem in a reasonable time for realistic applications using commercially available software and ordinary computers.

4) The proposed model is relevant to the operation of multiregional electricity markets such as those in the U.S.

\section{REFERENCES}

[1] A. L. Ott, "Experience with PJM market operation, system design, implementation," IEEE Trans. Power Syst., vol. 18, no. 2, pp. 528-534, May 2003.

[2] A. J. Conejo, E. Castillo, R. Minguez, and R. Garcia-Bertrand, Decomposition Techniques in Mathematical Programming. New York, NY, USA: Springer, 2006.

[3] F. Bouffard, F. D. Galiana, and A. J. Conejo, "Market-clearing with stochastic security-part I: Formulation," IEEE Trans. Power Syst., vol. 20, no. 4, pp. 1818-1826, Nov. 2005.

[4] F. Bouffard and F. D. Galiana, "Stochastic security for operations planning with significant wind power generation," IEEE Trans. Power Syst., vol. 23, no. 2, pp. 306-316, May 2008.

[5] J. M. Morales, A. J. Conejo, and J. Perez-Ruiz, "Economic valuation of reserves in power systems with high penetration of wind power," IEEE Trans. Power Syst., vol. 24, no. 2, pp. 900-910, May 2009.

[6] P. A. Ruiz, C. R. Philbrick, E. Zak, K. W. Cheung, and P. W. Sauer, "Uncertainty management in the unit commitment problem," IEEE Trans. Power Syst., vol. 24, no. 2, pp. 642-651, May 2009.

[7] A. Papavasiliou, S. S. Oren, and R. P. O'Neill, "Reserve requirements for wind power integration: A scenario-based stochastic programming framework," IEEE Trans. Power Syst., vol. 26, no. 4, pp. 2197-2206, Nov. 2011.

[8] A. Ahmadi Khatir and R. Cherkaoui, "Preventive and corrective security market model," in Proc. 17th Power Systems Computation Conf. (PSCC), Stockholm, Sweden, 2011.

[9] B. H. Kim and R. Baldick, "Coarse-grained distributed optimal power flow," IEEE Trans. Power Syst., vol. 12, no. 2, pp. 932-939, May 1997.

[10] R. Baldick, B. H. Kim, C. Chase, and Y. Luo, "A fast distributed implementation of optimal power flow," IEEE Trans. Power Syst., vol. 14, no. 3, pp. 858-864, Aug. 1999.

[11] A. J. Conejo and J. A. Aguado, "Multi-area coordinated decentralized DC optimal power flow," IEEE Trans. Power Syst., vol. 13, no. 4, pp. 1272-1278, Nov. 1998.

[12] F. J. Nogales, F. J. Prieto, and A. J. Conejo, "A decomposition methodology applied to the multi-area optimal power flow problem," Ann. Oper. Res., vol. 120, pp. 99-116, 2003.

[13] A. G. Bakirtzis and P. N. Biskas, "A decentralized solution to the DC-OPF of interconnected power systems," IEEE Trans. Power Syst., vol. 18, no. 3, pp. 1007-1013, Aug. 2003.

[14] A. Ahmadi-Khatir, A. J. Conejo, and R. Cherkaoui, "Multi-area energy and reserve dispatch under wind uncertainty and equipment failures," IEEE Trans. Power Syst., to be published.

[15] A. J. Conejo, F. J. Nogales, and F. J. Prieto, "A decomposition procedure based on approximate newton directions," Math. Program., vol. 93, pp. 495-515, 2002.
[16] J. M. Arroyo and A. J. Conejo, "Optimal response of a thermal unit to an electricity spot market," IEEE Trans. Power Syst., vol. 15, no. 3, pp. 1098-1104, Aug. 2000.

[17] J. Batut and A. Renaud, "Daily generation scheduling optimization with transmission constraints: A new class of algorithms," IEEE Trans. Power Syst., vol. 7, no. 3, pp. 982-989, Aug. 1992.

[18] G. Cohen, "Auxiliary problem principle and decomposition of optimization problems," J. Optimiz. Theory Applicat., vol. 32, no. 3, pp. 277-305, Nov. 1980.

[19] J. Eckstein, "Parallel alternating direction multiplier decomposition of convex programs," J. Optimiz. Theory Applicat., vol. 80, pp. 39-62, 1994.

[20] R. E. Rosenthal, GAMS-A User's Guide, GAMS Development Corp., Washington, DC, USA, 2010, tech. rep.

[21] Reliability Test System Task Force, "The IEEE reliability test system-1996," IEEE Trans. Power Syst, vol. 14, no. 3, pp. 1110-1120, Aug. 1999.

[22] Danish Minister for Climate and Energy. [Online]. Available: http:// energinet.dk.

[23] J. M. Morales, S. Pineda, A. J. Conejo, and M. Carrion, "Scenario reduction for futures market trading in electricity markets," IEEE Trans. Power Syst., vol. 24, no. 2, pp. 878-888, May 2009.

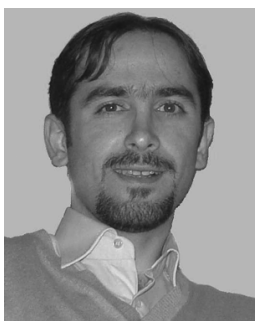

Ali Ahmadi-Khatir (S'09-M'13) received the M.Sc. degree from Sharif University of Technology, Tehran, Iran, in 2005, and the Ph.D. degree from EPFL, Lausanne, Switzerland, in 2013.

$\mathrm{He}$ is currently with the Ancillary Services Department of Swissgrid as a Research Engineer. His research interests include electricity markets, power system operation, and optimization.

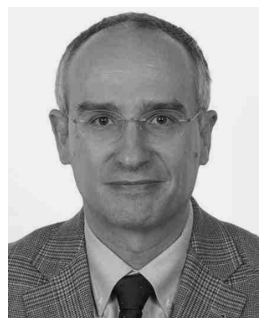

Antonio J. Conejo (F'04) received the M.S. degree from the Massachusetts Institute of Technology, Cambridge, MA, USA, in 1987, and the Ph.D. degree from the Royal Institute of Technology, Stockholm, Sweden, in 1990.

$\mathrm{He}$ is currently a full Professor at the University of Castilla-La Mancha, Ciudad Real, Spain. His research interests include control, operations, planning and economics of electric energy systems, as well as statistics and optimization theory and its applications.

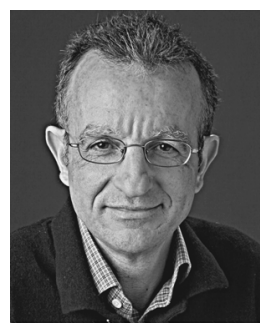

Rachid Cherkaoui (M'05-SM'07) received the M.S. and Ph.D. degrees from EPFL, Lausanne, Switzerland, in 1983 and 1992, respectively.

$\mathrm{He}$ is currently the head of the power system group at EPFL. His research interests include power system operation, planning, and electricity market. 